\title{
Study on Periplaneta americana (Blattodea: Blattidae) Fungal Infections in Hospital Sewer System, Esfahan City, Iran, 2017
}

\author{
Maryam Khodabandeh, ${ }^{1}$ Leila Shirani-Bidabadi, ${ }^{2}$ Mahboobe Madani, ${ }^{1}$ \\ and Alireza Zahraei-Ramazani $\mathbb{1}^{3}$ \\ ${ }^{1}$ Islamic Azad University Falavarjan Branch, Faculty of Microbiology, Department of Microbiology, University Boulevard, \\ Post Box: 155/84515, Falavarjan, Iran \\ ${ }^{2}$ Kerman University of Medical Sciences, School of Public Health, Department of Vector Biology and Control of Disease, \\ Haft-Bagh Highway, Post Box: 76169-13555, Kerman, Iran \\ ${ }^{3}$ Tehran University of Medical Sciences, School of Public Health, Department of Medical Entomology and Vector Control, \\ Enghelab Street, Post Box: 6446-14155, Tehran, Iran
}

Correspondence should be addressed to Alireza Zahraei-Ramazani; alirezazahraei@yahoo.com

Received 19 April 2020; Accepted 8 July 2020; Published 1 August 2020

Academic Editor: Patrizia Messi

Copyright (C) 2020 Maryam Khodabandeh et al. This is an open access article distributed under the Creative Commons Attribution License, which permits unrestricted use, distribution, and reproduction in any medium, provided the original work is properly cited.

\begin{abstract}
Background. American cockroaches contaminated with pathogens inside hospital manholes can be one of the major problems that health care systems face. Objectives. The aim of this study was to investigate the fungal infections of American cockroaches in the Esfahan hospital sewage network. The principle goal of the study was about the roaches as a vector of fungi and other pathogens. Method. The type of study was descriptive-analytical. A total of 55 American cockroach specimens from the manhole walls of the sewerage system of 7 large hospitals were captured. Samples were taken from the surface of the body, digestive tract, and haemocoel of cockroaches. The specimens were then cultured on Sabouraud dextrose agar separately, and fungi were identified according to the macroscopic and microscopic characteristics. Results. All cockroaches collected from hospitals were infected with fungi. Among the 24 (13 infected and 11 noninfected) (44\%) female cockroaches and 31 (18 infected and 13 noninfected) (56\%) male cockroaches, it was identified that $40.00 \%$ was infected with Aspergillus niger, $3.64 \%$ with Rhizopus, $7.27 \%$ with Penicillium, and 5.45\% with Mucor. 6 cockroaches had no yeast contamination. 17 (30.91\%) cockroaches were contaminated with Candida glabrata, 23 (41.82\%) cockroaches were contaminated with Candida krusei, and 22 (40\%) cockroaches were contaminated with other yeast species. The results of this study showed that Candida krusei had the highest prevalence among the isolated fungi with $35.37 \%$ of the digestive system and Aspergillus niger with $70.97 \%$ of the surface of the cockroach body. Conclusion. The results emphasized the role played by cockroaches as potential pathogenic vectors in hospital environments. Therefore, suitable management is needed for controlling this insect to prevent disease transmission in hospitals.
\end{abstract}

\section{Introduction}

Cockroaches are one of the oldest inhabitants of the Earth, dating back as far as the Carboniferous period, over 250 million yrs ago [1]. They lack special adaptations like the sucking mouthparts in some insects such as the aphids and other true bugs [2]. Over 4500 species of cockroaches have been identified, of which 40 species are associated with human habitats, while four species are well known as pests [3-5]. Cockroaches have chewing mouthparts and feed on a variety of materials (omnivorous), aiding in the mechanical transmission of various pathogenic viruses, bacteria, and protozoans to humans. Cockroaches have a worldwide distribution, especially in tropical and subtropical areas, and can tolerate a wide range of environments from the Arctic cold to tropical heat $[4,5]$. Their population increases in hot and humid places, especially with the availability of food and water [6].

Cockroaches feed indiscriminately on garbage and sewage and so have copious opportunities to disseminate 
human pathogens $[7,8]$. Also, their nocturnal and filthy habits make them ideal carriers of various pathogenic microorganisms [2].

Periplaneta americana (Linneaus, 1785) (Blattodea: Blattidae) are cosmopolitan cockroaches that occur frequently in urban sewage galleries and transit in anthropic environments, spreading pathogens; they are considered hazardous organisms to humans, causing serious health problems such as allergies, asthma, and others [9-12].

Periplaneta americana is the largest species of cockroaches found in Iran. It originated from South America, measures $30-40 \mathrm{~mm}$ in length, and is reddish-brown in color. The American cockroach lives in hot areas of buildings like the kitchens, heating rooms, warehouses, and sewage systems [4]. It usually comes out of its hiding places at night for feeding and other activities [13]. The adult cockroaches are long-lived and can live for as long as one year or more, producing a large number of egg capsules during this period, depending on food availability [14].

Sewerage is established to collect and convey sewage in urban areas in order to prevent contamination of soil and water resources. Some species of cockroaches including $P$. americana have colonized the sewerages, turning them into a suitable environment for reproduction and growth, posing serious health problems to humans [15].

Cockroaches are able to transfer fungi, bacteria, viruses, parasites, and other medically significant pathogenic agents on their body surfaces and in their feces in infectious regions, such as domestic habitats, hospitals, and industrial areas. From these insects collected from such environments, important pathogenic microorganisms have been isolated [13-18]. Plentiful pathogenic agents including 2 species of protozoans, 15 species of molds and fungi, 32 species of bacteria (such as Shigella and Salmonella), 1 virus, and 7 helminths which cause damage to humans are detected in the feces, in the gut, or on the cuticle of the cockroaches [19-21].

Hospitals, especially those with infectious diseases, have become the main areas for the transmission and spread of infections and a good place to grow and reproduce cockroaches, so that they can mechanically transmit those infectious agents and cause various diseases. Therefore, their struggle and control to create a safe and secure environment will achieve the basic goals of the hospitals. American cockroaches, due to specific biological behaviors, cause a variety of nosocomial and fungal infections at the hospital level and transmit and disperse these microorganisms in the environment and in the human places. Seven (external and internal) were found in the sewage network of seven hospitals with an infectious ward in Esfahan. The objective of our study was to investigate the fungal infections of American cockroaches in the Esfahan hospital sewage network.

\section{Materials and Methods}

2.1. Study Area. This research was carried out in the Esfahan city $\left(51^{\circ} 39^{\prime} 40^{\prime \prime} \mathrm{E} 32^{\circ} 38^{\prime} 30^{\prime \prime} \mathrm{N}\right)$ as a part of the central coordination of Esfahan, a central province of Iran. Esfahan is the capital city of the Esfahan province with an area of $250 \mathrm{~km}^{2}$ and 1695789 residents. It is the second most populous metropolitan city after Tehran in the central of Iran. Its altitude is $1571 \mathrm{~m}$, latitude $32^{\circ} 38^{\prime} 30^{\prime \prime} \mathrm{N}$, and longitude $51^{\circ} 39^{\prime} 40^{\prime \prime} \mathrm{E}$ (Figure 1). This city is located in a dry semidesert region with an annual rainfall of $102 \mathrm{~mm}$ and average summer maximum and winter minimum temperatures of $37.1^{\circ} \mathrm{C}$ and $-5.81^{\circ} \mathrm{C}$, respectively [22]. The Esfahan city has over 55375 manholes in its sewage system with an average depth of $9 \mathrm{~m}$ on the main lines and 1 meter on lateral lines. Most of the manhole covers were cast irons with two holes, and the average diameter of the holes was three centimeters. The shafts of the manholes were made of brick and cement coverings, and their stable moderate warmth and high humidity provide excellent conditions for cockroaches [23].

2.2. Collection Methods of Cockroaches. The sampling strategy of this study was the randomly clustered method from the internal sewage network manholes. Based on the sample size determination formula $n=p q z^{2} / d^{2} z=1.96$, $p=70 \% q=1-p=30 \% d=0.2$, the sample size calculated was $\sim 21$ samples. In this descriptive-analytical study, 55 American cockroaches randomly clustered were sampled from the internal sewage network manholes of 7 hospitals out of the 27 active hospitals in the urban area of the city of Esfahan, Iran, during 2016-2017, which necessarily had a large number of infectious, hospitalized, and traffic units. If there were not enough cockroaches in the first manhole, the second manhole was used. These 7 hospitals have been selected in such a way that their distribution indicates the entire city of Esfahan, i.e., they were selected in the north, south, east, and west, as well as the center of Esfahan.

Direct collection (hand capture) was used to collect the cockroaches. Cockroaches infected with white mycelium were slow-moving. The cockroaches were collected without being harmed. The name of the hospital and the exact location of the sample were noted on a label affixed to the containers. White moldy cockroaches were slower than other cockroaches. Each cockroach was placed in a single sterile test tube (falcon tube $15 \mathrm{ml}$ ), which was sterilized before, and transported to the laboratory at the Esfahan Health Research and Training Center, Tehran University of Medical Sciences, for identification and processing for fungi examination.

2.3. Isolation of Fungi from Cockroaches. In the laboratory, the cockroaches were immobilized by freezing at $0^{\circ} \mathrm{C}$ for $10 \mathrm{~min}$. Each anesthetized cockroach was examined under the dissecting microscope, and the species were identified using standard taxonomic keys. After identification, $2 \mathrm{ml}$ of sterile normal saline $(0.9 \% \mathrm{NaCl}$ salt $)$ was added to the test tube, and the cockroaches were vigorously shaken for $2 \mathrm{~min}$. After external washing, the cockroaches were washed with $70 \%$ ethyl alcohol for $2 \mathrm{~min}$. Then, the cockroaches were transferred to sterilized tubes and allowed to dry. Then, each cockroach was anesthetized with a chloroform-impregnated cotton pad and fixed on a work paper. The legs of the 


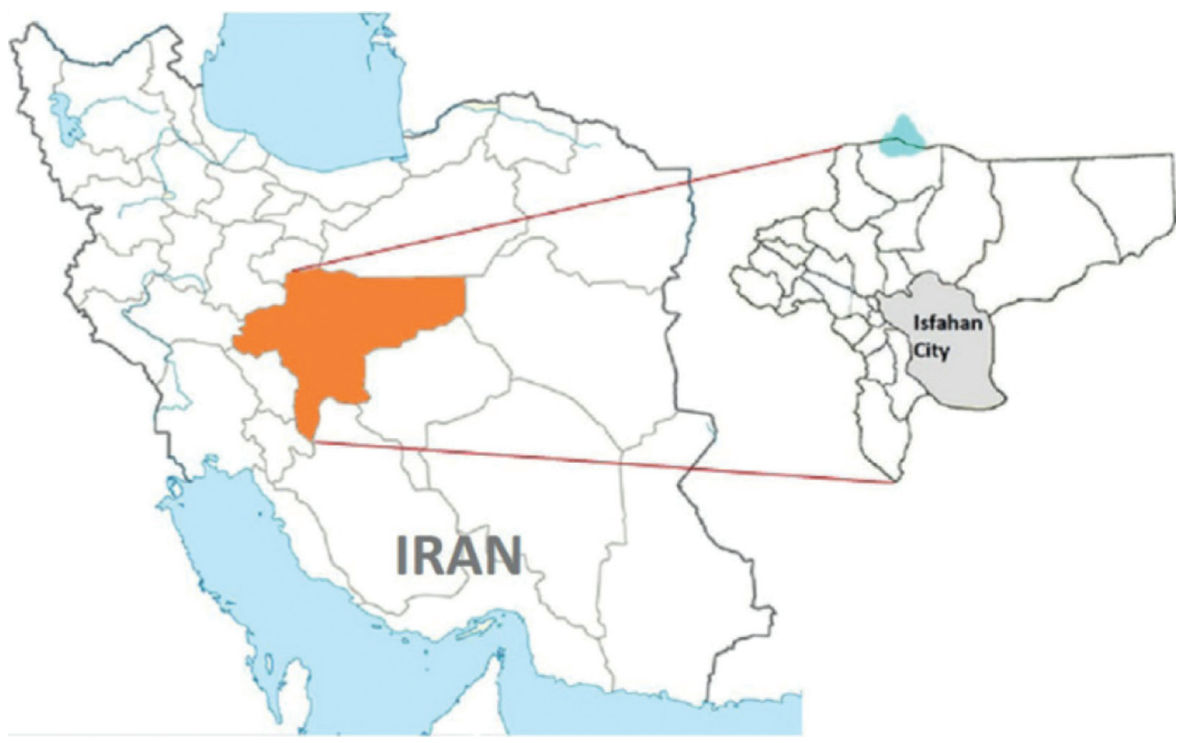

Figure 1: Study area of the Esfahan state and the city located in the central part of Iran, 2017 (prepared by the authors).

cockroach were fixed with a sterile needle to the work paper, and with the insect surgical scissors, the cockroaches were returned to the back of the body. The cockroaches were then washed twice in sterile normal saline for $3 \mathrm{~min}$ to remove traces of alcohol, and the gut was dissected out aseptically. The gut was then macerated under aseptic conditions in $2 \mathrm{ml}$ of sterile normal saline. The haemocoel portion of each cockroach was first homogenized, and the resulting mixture was put in a sterile container, and $2 \mathrm{ml}$ of physiological serum was added to it. The gastrointestinal tract and cockroach homogenate were individually placed in a microtube and homogenized by the vertex apparatus $[24,25]$.

2.4. Mycological Studies. The resulting macerate was cultured on Sabouraud's dextrose agar with $0.05 \%$ chloramphenicol and incubated at $37^{\circ} \mathrm{C}$ for 2 weeks. Suspensions from each cockroach section were centrifuged at $2000 \mathrm{rpm}$ for 10 minutes to perform mycological studies. Then, the outer and inner portions of the precipitate were cultured in $1 \mathrm{ml}$ in three separate plates containing Sabouraud dextrose agar with chloramphenicol. Two culture media were prepared from each sample, one incubated at $25^{\circ} \mathrm{C}$ and the other at $37^{\circ} \mathrm{C}$. Plates were kept for 7-14 days, and during this period, they were examined for fungal growth. The samples were then purified on the culture medium, and microscopic identification of the most frequent fungi was performed. Samples grown on Sabouraud dextrose agar were then purified. To identify different types of fungi, the macroscopic properties of fungal colonies, such as surface color and back of the colonies, colonial surface view of wrinkles, radial lines or concentric circles, and smoothness or creasing of the colonies, as well as the surface conditions of the colonies, such as powdery mildew, cotton, wool, velvety, and specificity, as well as microscopic characteristics were studied [26].

In order to identify yeasts, in addition to their appearance and colony characteristics that are of particular importance for diagnosis, their physiological properties, which are more important for their identification and determination, were used. To this end, the tubal mass or germ tube test and the Corn Meal Agar test were performed to investigate chlamydoconidia, as well as the Candida CHROMagar test [26].

2.5. Tubal Mass or Germ Tube Test. For C. albicans, the germ tube test was performed to differentiate between C. albicans and nonalbicans Candida (NAC). By using a sterile needle, one colony of pure yeast was added separately to the sterile tube containing $1 \mathrm{ml}$ of fresh human serum. The suspension was stored at $2^{\circ} \mathrm{C}$ for 1 to 2 hours. Then, a drop of the above suspension was examined under a microscope for the presence or absence of the germ tube. The formation of the germ tube more than twice the diameter of the yeast cell, the parallel wall, and the absence of troughs at the site of the germ tube formation confirm the presence of the yeast Candida albicans [27].

2.6. Chlamydoconidia Test on Corn Meal Agar with Tween 80 Culture. The colonies from CHROMagar and SDA were plated on the Corn Meal Agar with Tween 80 for morphological examination of the production of chlamydospores, blastospores, true hyphae, and branched pseudohyphae [27].

A linear culture was performed by using a sterile loop of each yeast colonies on the Corn Meal Agar medium containing $1 \%$ Tween 80 . The media were kept at $30^{\circ} \mathrm{C}$ for 72 hours and examined directly under a microscope at 24,48 , and 72 hours. Under these conditions, Candida albicans produces false hyphae, blastoconidia, and chlamydoconidia. Other species of Candida do not cause chlamydiosis [27].

2.7. Culture on CHROMagar Candida Medium. The CHROMagar Candida medium comprised peptone $(10 \mathrm{~g})$, glucose $(20 \mathrm{~g})$, agar (15 g), chloramphenicol $(0.5 \mathrm{~g})$, and 
chromogenic ix $(2 \mathrm{~g})$ per liter, $\mathrm{pH}$ 6.1. This medium was prepared according to the manufacturer's instructions and does not require autoclaving and is dispensed into the Petri plates after cooling. The culture was inoculated, and incubation was done at $37^{\circ} \mathrm{C}$. The appearance of colonies, including color, size, and textures on the CHROMagar Candida medium, was analyzed [27].

All yeasts isolated from cockroaches were cultured on a CHROMagar company medium (CHROMagar company, Paris, France) and kept at $35^{\circ} \mathrm{C}$ for 48 hours. Based on the specific color created in the culture medium, species identification was performed. This medium is valid for the identification of the yeasts of Candida albicans, Candida tropicalis, and Candida krusei. These yeasts in this medium create a colony of green, gray, and pink colors. The culture on the chromium agar medium of Candida is valuable because of its ease of use, its ability to isolate Candida from clinical specimens, and the ability to identify up to several Candida species in the clinical specimen at a time [27].

Potential limitation of this study included the following: (1) collection of cockroaches from manholes of sewage, (2) isolation and culture of fungi, and (3) the PCR method due to high prices and shortage of materials due to sanctions in Iran.

\section{Results}

A total of 55 American cockroaches, Periplaneta americana, were sampled and studied from the sewage manholes of studied hospitals in Esfahan. Among them were 24 (13 infected and 11 noninfected) (44\%) female cockroaches and 31 (18 infected and 13 noninfected) (56\%) male cockroaches. The results of the cumulative frequency distribution of different fungal species isolated on the basis of fungal isolation from 55 American cockroaches are presented in Table 1 .

All cockroach samples were infected with 8 different fungal species. $35.37 \%$ of the contamination was related to Candida krusei and $70.97 \%$ to Aspergillus niger. Both mold and yeast were isolated from American cockroaches. Out of the 31 male cockroaches, 15 were infected with Aspergillus niger, 1 with Penicillium, 1 with Rhizopus, 1 with Mucor, and 13 with no infection. Out of the 24 female cockroaches, 7 were infected with Aspergillus niger, 3 with Penicillium, 1 with Rhizopus, 2 with Mucor, and 11 with no infection.

In relation to yeast fungi in male cockroaches, 10 cases of Candida glabrata infection, 15 cases of Candida krusei infection, 16 cases of Candida kluyveri infection, 5 cases of Candida viswanathii infection, and 5 cases of no contamination were reported.

Concerning the female sex of cockroaches, 11 cases of Candida glabrata infection, 14 cases of Candida kudriavzevii infection, 8 cases of Candida kluyveri infection, 3 cases of Candida viswanathii infection, and 2 cases of noncontamination were reported (Table 2). There was no significant difference in the abundance of yeast fungi in male and female cockroaches, such as mold fungi.
Based on macroscopic and microscopic observations, 24 cockroaches (43.63\%) were not infected with mold, 22 (40\%) were infected with Aspergillus niger, 2 (3.64\%) were infected with Rhizopus, 4 (7.27\%) were infected with Penicillium, and 3 (5.45\%) were infected with Mucor.

None of the yeasts isolated from cockroaches were Candida albicans, according to the results of germ tube testing, Corn Meal Agar, and chromium agar. Based on the specific color created in the chromium agar Candida medium, the identification of yeast species was performed. Colonies were created in green, gray, and pink, confirming the yeasts of Candida albicans, Candida tropicalis, and Candida krusei [26]. Large pink-to-purple yeast colonies of Candida glabrata were reported [27].

The results of the frequency distribution of yeast fungi isolated from cockroaches showed that 6 cockroaches had no yeast fungal contamination. Also, 17 (30.91\%) were infected with Candida glabrata, 23 (41.82) with Candida kudriavzevii, and $22(40 \%)$ were contaminated with other mold (Table 2).

\section{Discussion}

The main objective of this study was to examine human pathogenic microorganisms (bacteria and fungi) on the external and internal body parts of the American cockroach (Periplaneta americana) from the hospital sewer system in Esfahan city, Iran. American cockroach was collected from different manholes of the sewer system. This study showed that American cockroach is one of the dominant and active cockroaches in Esfahan health centers.

Different pathogenic and nonpathogenic fungal agents were recovered from this cockroach in human environments. Periplaneta americana can carry pathogenic fungi in its internal organs. Therefore, $P$. americana is much more than a harassment, and it has important health hazards as a mechanical vector. Therefore, the abundance of American cockroaches' population has to be reduced by various control methods, such as the proper management of garbage and organic waste disposal, sanitation, and using safe insecticides. Clearly, the presence of cockroaches in sensitive environments, hospitals, and houses is more dangerous than other parts due to the special circumstances and the special people hospitalized and can affect the environment, people, and community health. The density of cockroaches in most parts of the hospital and residential dwellings, their feeding from secretions and human feces, and their ability to transmit a wide range of pathogenic agents make them an ideal vector to transmit most medically important microorganisms. Nowadays, cockroaches have access to an infection source, human food, and the place for food production, and their role in the transmission of the disease is undeniable. Dehghani et al. studied cockroach fauna and frequency in human residential habitats in the north of Esfahan, in the Shahin Shahr city [28]. 675 of 1000 studied houses $(67.5 \%)$ were infested by all life stages of the cockroaches, and $32.5 \%$ had no infestation. $46 \%$ of infested houses had few, 30\% had medium, and 24\% had high infestation. The bathrooms and toilets were recognized as the 
TABLE 1: The cumulative frequency distribution of different fungal species isolated on the basis of fungal isolation from 55 American cockroaches, Esfahan, Iran, 2016-2017.

\begin{tabular}{|c|c|c|c|}
\hline \multirow[t]{2}{*}{ Row } & \multirow[t]{2}{*}{ Fungi species } & \multicolumn{2}{|c|}{$\begin{array}{l}\text { American cockroach } \\
\text { (number + percentage) }\end{array}$} \\
\hline & & Number & Percentage \\
\hline 1 & Aspergillus niger & 22 & 23.66 \\
\hline 2 & Rhizopus & 2 & 2.15 \\
\hline 3 & Penicillium & 4 & 4.30 \\
\hline 4 & Mucor & 3 & 3.22 \\
\hline 5 & Candida glabrata & 17 & 18.28 \\
\hline 6 & Candida krusei (Candida kudriavzevii) & 23 & 24.73 \\
\hline 7 & Yeast (other yeast) (Candida viswanathii + Candida kluyveri) & 22 & 23.66 \\
\hline 8 & Total number & 93 & 100 \\
\hline
\end{tabular}

TABLE 2: The cumulative frequency distribution of different fungal species isolated on the basis of fungal isolation from 55 American cockroaches collected from hospital sewage, Esfahan, Iran, 2016-2017.

\begin{tabular}{|c|c|c|c|c|c|c|}
\hline \multirow[b]{2}{*}{ Row } & \multirow[b]{2}{*}{ Fungi isolated } & \multicolumn{3}{|c|}{ Place of fungi isolated from cockroaches } & \multicolumn{2}{|c|}{ Total } \\
\hline & & $\begin{array}{c}\text { Exterior surface no. } \\
(\%)\end{array}$ & $\begin{array}{c}\text { Digestive system no. } \\
(\%)\end{array}$ & $\begin{array}{c}\text { Haemocoel no. } \\
(\%)\end{array}$ & Numb & Per \\
\hline 1 & Aspergillus niger & $11(50)$ & $10(45.45)$ & $1(4.55)$ & 22 & 100 \\
\hline 2 & Rhizopus & $2(100)$ & - & - & 2 & 100 \\
\hline 3 & Penicillium & $3(75)$ & $1(25)$ & - & 4 & 100 \\
\hline 4 & Mucor & $3(100)$ & - & - & 3 & 100 \\
\hline 5 & Candida glabrata & $7(41.18)$ & $9(52.94)$ & $1(5.88)$ & 17 & 100 \\
\hline 6 & Candida krusei (Candida kudriavzevii) & $9(39.13)$ & $12(52.17)$ & $2(8.70)$ & 23 & 100 \\
\hline 7 & $\begin{array}{c}\text { Yeast (other yeast) (Candida viswanathii }+ \text { Candida } \\
\text { kluyveri) }\end{array}$ & $6(27.27)$ & $11(50)$ & $5(22.73)$ & 22 & 100 \\
\hline & Total number & 41 & 43 & 9 & 93 & 100 \\
\hline
\end{tabular}

most infested places (41\%) [28]. The propensity of American cockroaches to move freely and dwell in sewers, restrooms, and drains make the problem worse. The ability of cockroaches in the transmission of pathogens is emphasized in many types of research in this regard. Infectious agents carried by cockroaches can infect humans, animals, and food resources in some conditions $[29,30]$. This study confirmed that these insects in residential areas were contaminated with fungi of medical importance. A total of 8 different fungal species were isolated from American cockroaches. In this study, a high number of the cockroach specimens (55 number) from the sewer system were found to carry known fungal pathogens including Rhizopus sp., Mucor sp., Penicillium spp., Candida spp., and Aspergillus spp. Thus, the isolation of medically important fungi suggests a serious risk concern for patients. Although the direct involvement of American cockroaches in the transmission of infectious agents is difficult to demonstrate, several other studies have also isolated, from cockroaches from residential areas and hospitals, medically important fungi [24, 31, 32].

In the present study, all cockroach samples were infected with 8 different fungal species. $35.37 \%$ of the contamination was related to Candida krusei and $70.97 \%$ to Aspergillus niger. Both mold and yeast were isolated from American cockroaches. Out of the 31 male cockroaches, 15 were infected with Aspergillus niger, 1 with Penicillium, 1 with Rhizopus, 1 with Mucor, and 13 with no infection. Out of the 24 female cockroaches, 7 were infected with Aspergillus niger, 3 with Penicillium, 1 with Rhizopus, 2 with Mucor, and 11 with no infection. The findings from this study about medically important fungi isolated from cockroaches agree with the results of some researchers. In a study in Thailand, Penicillium spp. and Aspergillus spp. appeared frequently on the integument of $16(35.6 \%)$ and 11 (24.4\%) cockroaches, respectively [32]. In another study in Brazil, Candida sp. (38.6\%), Aspergillus sp. (30.7\%), and Penicillium sp. (8.9\%) were the most common fungi recovered on cockroaches [31]. In addition, in Sari (Iran), Candida spp., Aspergillus spp., and Rhodotorula spp. were the most common fungi that appeared on the cuticle of cockroaches [30]. Kasseri et al. [33] in the Khuzestan province, Ahvaz city, showed the fungi isolated from the digestive system of Periplaneta americana (Blattaria: Blattidae) trapped from residential dwellings [33]. Results of their study showed that a high percentage of cockroaches (88.6\%) were detected to carry fungi of medical importance. Overall, 23 fungi species/genera were isolated from the American cockroaches' alimentary tract. The fungi isolated from cockroaches from the residential regions were species of Aspergillus, Rhizopus, Penicillium, Mucorales, Alternaria, Cladosporium, Mycelia, Chrysosporium, Candida, Rhodotorula, Zygosaccharomyces, and Debaryomyces. Candida spp. (41.4\%), Aspergillus spp. (37.1\%), and Rhodotorula spp. $(27.1 \%)$ were the most common fungi recovered on cockroaches. Candida albicans and Candida glabrata were the commonest species of the genus Candida. In addition, 
Aspergillus niger and A. flavus were the most frequent species of the genus Aspergillus [33].

In a survey in Kashan (Iran) by Droodgar in 2004, the prevalence of fungal agents in cockroaches was $41.1 \%$ and $22.8 \%$, respectively. Candida spp. (39.5\%), Aspergillus spp. (37. 2\%), and Penicillium spp. (5.4\%) had a maximum prevalence among the fungi observed [34]. In India, Candida spp. and Aspergillus spp. were the most frequent fungi of medically important genera from cockroaches from a hospital and a residential area [35]. The finding of the present study also showed that Aspergillus niger (70.97\%) was the most common species isolated from cockroaches. Moreover, A. flavus and A. fumigatus were the most frequently recovered species from cockroaches [31]. Aspergillus niger was significantly more frequent in the residential area and the hospital [24]. In a study in the hospital environments in the Ahvaz city, 28 fungal species were isolated from adult houseflies. The main fungi isolated were Aspergillus spp. (67.4\%), Penicillium sp. (11.6\%), Mucorales sp. (11\%), Candida spp. (10.5\%), and Rhodotorula sp. (8.4\%) [35].

In the present study, from the external surface of the cockroaches studied, Aspergillus niger, Penicillium, Mucor, and Rhizopus molds, from the cockroach, Aspergillus niger molds, and from the gastrointestinal tract of cockroaches, Aspergillus niger and Penicillium, were isolated. Candida glabrata were isolated from the haemocoel, the gastrointestinal tract, and the outer surface, respectively, and 2,12, and 9 cases of Candida krusei were also isolated, respectively. The chromium agar assay was used for the identification of Candida albicans, Candida tropicalis, and Candida krusei, and other yeasts isolated by the chromium agar method belonged to other Candida species or other yeasts. Mirhendi and colleagues identified candidate strains isolated from patient samples and standard strains of Candida by culturing on the Candida chromium agar medium and showed that the results were in good agreement with the PCR_RFLP molecular assay [26].

We have displayed that American cockroaches transport a great number of species of medically significant fungi in their digestive system, incriminated as significant agents in nosocomial infections. Hospital-acquired fungal infections are considered consequential causes of morbidity in immune-compromised individuals, especially those remaining in the hospital for a long period [36]. The results of the 2003 phelan study showed that the German cockroach also played a very important role in the transmission of pathogens in hospitals [37]. Cockroaches living near human environments were significant vectors of etiological agents and all groups of possible pathogens such as protozoans, bacteria, helminths, and viruses. Various bacteria universally associated with these insects are recognized to inure diarrhea, dysentery, and food intoxication in humans. In the present study, Aspergillus niger and four species of Candida (Candida kudriavzevii, Candida kluyveri, Candida glabrata, and Candida viswanathii) were isolated from cockroaches. Aspergillus niger molds were isolated from the studied strains alone, and Aspergillus niger and Penicillium were isolated from their gastrointestinal tract. Finally, all four Candida species were isolated from the haemocoel and the gastrointestinal tract and the outer surface of the cockroaches. As mentioned, different species of fungi in this study and other similar studies have been isolated from cockroaches. In the present study, for the first time, four yeast strains (Candida kudriavzevii, Candida kluyveri, Candida glabrata, and Candida viswanathii) not isolated in previous studies were isolated. It is certain that a high contamination of cockroaches with pathogens can cause fungal epidemics in hospitals. Given the importance of pathogenic fungi in cockroaches, paying attention to public health and the need to control different ways of controlling them in our hospitals and medical centers are necessary [30]. Each hospital has a good prognosis for the fungal diseases in that hospital and the surrounding areas, which are important for the timely diagnosis, treatment, and control of patients. Since all of these hospitals had infectious wards, occasional reports of infection sent to researchers. Sometimes, a lot of people from the city are infected with these pathogens, and especially, they lived around these hospitals. So, the selection of these hospitals and sampling of American cockroaches can be generalized to the whole city. Of course, the results of this research will make people and officials be aware and will make it easier and more practical to control these disease vectors. Zahraei-Ramazani et al. [23] used various chemical and physical methods to control American cockroaches (Periplaneta americana) inside manholes of the municipal sewage disposal system in Esfahan city, Central Iran, and Esfahan's urban internetwork [23]. Their results showed that almost all of the products (excluding boric acid with bait formulation) resulted in appropriate control within one month of application. The appropriate products for chemical control of cockroaches were chlorpyrifos 5\% emulsifiable concentrate (EC), diazinon 5\% (EC), diazinon $0.05 \%$ (EC), and cypermethrin $5 \%$ fog. These pesticides achieved an optimal reduction of population, providing more than $90 \%$ control of cockroaches for five consecutive months. They concluded that the emulsifiable concentrates and fog formulations were more successful compared to other methods, as they can penetrate deep into the hiding places of cockroaches [23].

Therefore, factors such as the spread of fungal diseases in the city and the dispersal of hospitals, especially hospitals with infectious diseases, as well as the equipping of cities with a sewage sanitation network and related manholes that are installed and dispersed for inspection inside the municipal sewage disposal system, move cockroaches from hospitals to residential and public places and transmit fungal diseases. Therefore, performing various methods to control cockroaches in the urban space network before the invasion of cockroaches from inside the manholes into places and doing and observing hygienic points by people that do not prevent cockroaches from entering their residential areas are recommended. And, the control of cockroaches in hospitals is especially recommended.

\section{Conclusion}

In this study, yeasts were isolated from American cockroaches in Esfahan. High contamination of cockroaches with pathogens can cause fungal epidemics in hospitals and in the surrounding areas. Based on the results of this study, 
Aspergillus niger, Penicillium, Mucor, Rhizopus, Candida glabrata, and Candida krusei molds were isolated from Esfahan cockroaches. Of course, the results of this research will make people and officials be aware and will make it easier and more practical to control these diseases. Due to the importance of the pathogenicity of the abovementioned fungi, it is necessary to pay attention to public health and to eliminate these insects. On the other hand, awareness of the common fungi in each region and each hospital provides a good prognosis for fungal diseases in that hospital, which is important for timely diagnosis and treatment of patients.

\section{Data Availability}

The data used to support the findings of this study are available from the corresponding author upon request.

\section{Conflicts of Interest}

The authors declare that they have no conflicts of interest.

\section{Authors' Contributions}

M. Kh. and M. M contributed to the study design, data analysis, and interpretation and provided approval of the final version of the manuscript to be published. L. SH. B. and A. Z. R. contributed to the data collection, preparation, revision, and manuscript writing and revision.

\section{Acknowledgments}

This study was conducted jointly between the Esfahan Health Research Station affiliated to the Tehran University of Medical Sciences and Islamic Azad University. Therefore, the authors of the article from the Islamic Azad University Research Council, Falavarjan Branch, approved this project as a Master's student thesis. Thanks are due to the field of microbiology. This study was financially supported (numbering project: 17230507942003) by the Islamic Azad University Research Council, Falavarjan Branch.

\section{References}

[1] M. R. Fakoorziba, F. Eghbal, J. Hassanzadeh, and M. D. Moemenbellah-Fard, "Cockroaches (PeriplanetaamericanaandBlattellagermanica) as potential vectors of the pathogenic bacteria found in nosocomial infections," Annals of Tropical Medicine \& Parasitology, vol. 104, no. 6, pp. 521-528, 2010.

[2] A Salehzadeh, P. Tavacol and H. Mahjub, "Bacterial, fungal and parasitic contamination of cockroaches in public hospitals of Hamadan," Journal of Vector Borne Diseases, vol. 44, pp. 105-110, 2007.

[3] X. Bonnefoy, H. Kampen, and K. Sweeney, Public Health Significance of Urban Pests, World Health Organization, Copenhagen, Denmark, 2008.

[4] D. L. Wannigama, R. Dwivedi, and A. R. Zahraei-Ramazani, "Prevalence and antibiotic resistance of gram-negative pathogenic bacteria species isolated from Periplaneta americana and Blattella germanica in Varanasi, India," Journal of Arthropod-Borne Diseases, vol. 8, pp. 10-20, 2014.
[5] S. S. Hashemi-Aghdam and M. A. Oshaghi, "A checklist of Iranian cockroaches (Blattodea) with description of Polyphaga sp. as a new species in Iran," Journal of ArthropodBorne Diseases, vol. 9, pp. 161-175, 2015.

[6] A. Hanafi-Bojd and S. Sadaghiani, Iran Cockroaches, Tehran University of Medical Sciences Publishers, Tehran, Iran, 1st edition, 2001.

[7] M. F. Cotton, E. Wasserman, C. H. Pieper et al., "Invasive disease due to extended spectrum beta-lactamase-producing Klebsiella pneumoniae in a neonatal unit: the possible role of cockroaches," Journal of Hospital Infection, vol. 44, no. 1, pp. 13-17, 2000.

[8] H.-H. Barnes, W.-C. Chen, and C.-F. Peng, "Isolation of bacteria with antibiotic resistance from household cockroaches (Periplaneta americana and Blattella germanica)," Acta Tropica, vol. 93, no. 3, pp. 259-265, 2005.

[9] B. W. Allen, "Excretion of viable tubercle bacilli by Blatta orientalis (the oriental cockroach) following ingestion of heatfixed sputum smears: a laboratory investigation," Transactions of the Royal Society of Tropical Medicine and Hygiene, vol. 81, no. 1, pp. 98-99, 1987.

[10] P. J. Thyssen, T. d. C. Moretti, M. T. Ueta, and O. B. Ribeiro, "O papel de insetos (Blattodea, Diptera e Hymenoptera) como possíveis vetores mecânicos de helmintos em ambiente domiciliar e peridomiciliar," Cadernos de Saúde Pública, vol. 20, no. 4, pp. 1096-1102, 2004.

[11] A. S. Pinto, M. M. Rossi, E. Salmeron, and C. S. Pinto, "O meio urbano e as pragas urbanas," in de pragas urbanas, A. S. Pinto, M. M. Rossi, and E. M. Salmeron, Eds., Piracicaba, Brazil, 2007.

[12] H. Kassiri and S. Kazemi, "Cockroaches (Periplaneta americana (L.), dictyoptera:blattidae) as carriers of bacterial pathogens, khorramshahr county, Iran," Jundishapur Journal of Microbiology, vol. 5, pp. 320-322, 2012.

[13] W. J. Bell, L. M. Roth, and C. A. Nalepa, Cockroaches: Ecology, Behavior, and Natural History, Microbes: Unseen Influence, pp. 76-88, The Johns Hopkins University Publishers, Baltimore, Maryland, 2007.

[14] C. A. Strong, S. M. Valles, P. G. Koehler, and R. J. Brenner, "Residual efficacy of Blatticides applied to surfaces contaminated with German cockroach (Dictyoptera: blattellidae) feces," The Florida Entomologist, vol. 83, no. 4, pp. 438-445, 2000.

[15] M. W. Service, A Guide to Medical Entomology, p. 110, 1st edition, Tehran University of Medical Sciences Publishers, Tehran, Iran, 1980.

[16] M. H. Feizhaddad, H. Kassiri, and M. R. Sepand, "Ghasemi Bacteriological survey of American cockroaches in hospitals," Middle-East Journal of Scientific Research, vol. 12, no. 7, pp. 985-989, 2012.

[17] H. Kassiri, A. Kasiri, and A. Quaderi, "Detection and prevalence rate of American cockroaches (Periplaneta americana) bacterial infections in human dwellings," Archives of Clinical Infectious Diseases, vol. 9, no. 4, pp. 1-3, 2014.

[18] H. Kassiri, A. Kassiri, and S. Kazemi, "Investigation on American cockroaches medically important bacteria in Khorramshahr hospital, Iran," Asian Pacific Journal of Tropical Disease, vol. 4, no. 3, pp. 201-203, 2014.

[19] A. A. K. Kazemi and H. Vatani, "A survey on species and prevalence rate of bacterial agents isolated from cockroaches in three hospitals," Vector-Borne and Zoonotic Diseases, vol. 9, no. 2, pp. 197-200, 2009.

[20] J. Allotey, S. Mpuchane, B. A. Gashe, M. Simpanya, and I. Matsheka, "Trapping of Blattella germanica (L) populations 
in human dwellings in Gaborone, Botswana," Journal of Zoo and Aquarium Research, vol. 20, no. 2, pp. 175-188, 2009.

[21] S. Akbari, M. A. Oshaghi, S. S. Hashemi-Aghdam, S. Hajikhani, G. Oshaghi, and M. H. Shirazi, "Aerobic bacterial community of American cockroach Periplaneta americana, a step toward finding suitable paratransgenesis candidates," Journal of Arthropod-Borne Diseases, vol. 9, no. 1, pp. 35-48, 2015.

[22] H. Varesi, H. Beikmohammadi, and S. Ghanbari, "Compassion of economic dam-ages of Agricultural drought in Naein City with other townships in Esfahan (for 1378-1382 years)," Journal of Environmental Planning and Management, vol. 21, pp. 21-44, 2010.

[23] A. R. Zahraei-Ramazani, A. Saghafipour, and H. Vatandoost, "Control of American cockroach (Periplaneta americana) in municipal sewage disposal system, Central Iran," Journal of Arthropod-Borne Diseases, vol. 12, no. 2, pp. 172-179, 2018.

[24] S. Chitsazi, G. H. Moravvej, and M. Naderi-Nasab, "A survey on the bacterial and fungal contamination of German cockroaches in Mashhad Imam- Reza hospital during 20092010," FEYZ, vol. 16, no. 6, pp. 576-584, 2013.

[25] S. Chitsazi, G. Moravvej, and M. Naderinasab, "Fauna of bacteria and fungi associated with digestive system of Blattela germanica L. collected from various locations in Mashhad, Iran," Iranian Journal of Microbiology, vol. 6, no. 4, pp. 16-26, 2013.

[26] H. Mirhendi, K. Makimura, M. R. Shidfar, and L. Hosinpor, "Identification and frequency of Candida species isolated from patients using chromic agar Candida," Scientific Journal of Hamadan University of Medical Sciences and Health Services, vol. 13, no. 4, pp. 11-15, 2006.

[27] S. G. Nadeem, S. T. Hakim, and S. U. Kazmi, "Use of CHROM agar Candida for the presumptive identification of Candida species directly from clinical specimens in resource-limited setting," Libyan Journal of Medicine, vol. 5, 2010.

[28] R. Dehghani, M. Atharizadeh, S. G. Moosavi, S. Azadi, M. Rashidi, and A. Paksa, "Analysis of cockroach fauna and frequency in human residential habitats of north of isfahan, Iran ISSN: 2383-2568," Quarterly of International Archives of Health Sciences, vol. 1, no. 1, p. 25, 2014

[29] A. Vahabi, K. Shemshadn, P. Mohammadi, M. Sayyadi, M. Shemshad, and J. Rafinejad, "Microbiological study of domestic cockroaches in human dwelling localities," African Journal of Microbiology Research, vol. 5, pp. 5790-5792, 2011.

[30] S. F. Motevali Haghi, S. R. Aghili, S. Gholami et al., "Isolation of medically important fungi from cockroaches trapped at hos-pitals of Sari, Iran," Bulletin of Environment, Pharmacology and Life Sciences, vol. 3, no. 5, pp. 29-36, 2014.

[31] A. A. Lemos, J. A. Lemos, M. A. Prado et al., "Cockroaches as carriers of fungi of medical importance," Mycoses, vol. 49, no. 5, pp. 23-25, 2006.

[32] P. Saichua, K. Pinmai, S. Somrithipol, and S. Tor-Udom, "Isolation of medically important fungi from cockroaches trapped at Thammasat Chalermprakiat Hospital," Thammasat Medical Journal, vol. 8, no. 3, pp. 345-351, 2008.

[33] H. Kassiri, M. Zarrin, and R. Veys-Behbahani, "Pathogenic fungal species associated with digestive system of Periplaneta americana (blattaria: Blattidae) trapped from residential dwellings in Ahvaz city, southwestern Iran," Journal of Arthropod-Borne Diseases, vol. 12, no. 1, pp. 16-23, 2018.

[34] A. Doroodgar, M. Arbabi, and M. A. Asadi, "Survey on parasitic and fungal agents of hospital cockroaches in Kashan2002," FEYZ, vol. 10, no. 1, pp. 28-35, 2006, In Persian.
[35] H. Kassiri, M. Zarrin, R. Veys-Behbahani, S. Faramarzi, and A. Kasiri, "Isolation and identification of pathogenic filamentous fungi and yeasts from adult house fly (Diptera: muscidae) captured from the hospital environments in Ahvaz city, southwestern Iran: table 1," Journal of Medical Entomology, vol. 52, no. 6, pp. 1351-1356, 2015.

[36] M. M. McNeil, S. L. Nash, R. A. Hajjeh et al., "Trends in mortality due to invasive mycotic diseases in the United States, 1980-1997," Clinical Infectious Diseases, vol. 33, no. 5, pp. 641-647, 2001

[37] K. Phelan, H. Ladonni, H. Asle Soleimani, S. H. Afhami, and M. Shayeghi, "The role of German Cockroach in hospital infections," Journal of School of Public Health and Institute of Public Health Research, vol. 2, pp. 43-53, 2003. 